\title{
A NEW APPROACH TO OPTIMIZING NETWORK BANDWIDTH AND TRANSFER RATE ON MISSION CRITICAL COMPUTER NETWORKS: NETWORK QUALITY AND RESOURCE MANAGER
}

\author{
Kerem ERZURUMLU ${ }^{1 *}$ \\ ${ }^{1}$ Ordu University, Department of Computer Programming, 52200 Ordu, Turkey
}

Received: March 16, 2020; Accepted: March 20, 2020; Published: April 01, 2020

\begin{abstract}
In this paper, a new approach and solution of the one of the biggest problem on mission critical networks, network and bandwidth optimization will be described. The developed "Network Quality and Resource Manager" ensures the quality of network bandwidth and transfer rate on isolated mission critical networks like high resolution IP based security camera surveillance systems. The "Network Quality and Resource Manager" uses "Quality of Service", and "Address Resolution Protocol Table" via Simple Network Management Protocol (a.k.a. SNMP) over manage-able at-least 2nd layer network devices. Hence NQRM uses SNMP, it is vendor independent and can work with any network architecture.
\end{abstract}

Keywords: Resource manager, SNMP, Quality of service, ARP table, Mission critical networks

*Corresponding author: Ordu University, Department of Computer Programming, 52200 Ordu, Turkey

E mail: kerem@linux.org.tr (K. ERZURUMLU)

Kerem ERZURUMLU (iD) https://orcid.org/0000-0001-5363-1963

Cite as: Erzurumlu K. 2020. A new approach to optimizing network bandwidth and transfer rate on mission critical computer networks: Network quality and resource manager. BSJ Eng Sci, 3(2): 57-59.

\section{Introduction}

With the rapidly developing technology on digital processing and high speed networks, most of the conventional analog operations are becoming digital. Digital technology and operations achieve more secure, reliable and accurate results on desired implementation. Once the operations became digital, most of the time -if a distance involved in the procedure-, digital tools communicate with its server/controller via Internet Protocol (a.k.a. IP), Transmission Control Protocol/User Datagram Protocol (a.k.a. TCP/UDP) and Ethernet technology.

But the Ethernet technology has its own disadvantages. In most cases, network devices latencies, queue overloads, delays of Address Resolution Protocol requests/replies, interrupts the communication between the server and device.

When the operations are mission/data critical, the communication between the server and device cannot be interrupted or delayed for any reason. A good example for this type of communication will be security camera video feeds. Those feeds network requirements are constant. Any interruption/delaying of these feeds causes analytic software errors and recording issues. In some of these cases, the video which has been recorded from the security camera becomes corrupted.

The "Network Quality and Resource Manager" software which will be explained within this paper targets only networks which has multiple purposes simultaneously. 
The main goal to create and establish a stable and durable connection between the server and device using Quality of Service (a.k.a. QoS) and preset Address Resolution Protocol (a.k.a. ARP) table entries.

In order to gain these stable connection, the network elements which is in the path between device and server must support Quality of Service and Simple Network Management Protocol.

Address Resolution Protocol: In order to communicate between two devices in Ethernet Protocol which are located on same network segment, they both must learn the recipient's Media Access Control (a.k.a. MAC) addresses. This "learning" process is done via Address Resolution Protocol Request and Address Resolution Protocol Reply. The initializer of the communication broadcast an Address Resolution Protocol Request package. Hence the package is broadcasted, all the devices which are connected to this network receives the package. But only the interested communication partner replies this package in unicast. (Dostálek and Kabelová, 2016)

Quality of Service: Most of the time, Quality of Service is used with Voice Over Internet Protocol (VoIP). Hence the voice packages are time related and critical packages, in a network environment, these packages must be handled prior to other packages. For this queue correction, network devices comes with a feature called Quality of Service. This features guarantees the described packages handled accord. (Prasad and Prasad, 2005).

\section{Material and Method}

In the starting process of Network Quality and Resource Manager, the Manager firstly scans the previously registered network devices. If a network device is up and running, Manager communicates with it via Simple Network Management Protocol and obtains its routing table records. When all the devices in the Manager's list completed, Manager will know the network architecture and paths between the network devices. Then the Manager enters to idle mode to wait for incoming requests.

When a server application needs a stable and durable connection with a device, first it makes a request in XML format to the Manager. A sample Manager Request and these request's response are given in Figure 1 and Figure 2. The Manager handles the request and determine the server and client IP addresses. Then Manager detects the each IP Addresses network IDs and their locations on network architecture. Then the shortest path between the systems are calculated.

When the path is calculated, all the nodes of the path (including the end nodes) are connected via Simple Network Management Protocol. Both the end nodes Address Resolution Protocol Tables are the first ones to update. The Media Access Control Address of the router/third layer switch which is responsible from the server's network is added/updated in the server's
Address Resolution Protocol Table. Then a same procedure is done for client. If there are switches between the router and server/client, their Port Address Table is also updated.

\begin{tabular}{|c|}
\hline $\begin{array}{l}<\text { ?xml version="1.0"? }> \\
<\text { ManagerRequest }> \\
\quad<\text { Version }>1.0</ \text { Version }> \\
\quad<\text { Action }>0</ \text { Action }> \\
\quad<\text { Source }>192.168 .1 .100</ \text { Source }> \\
\quad<\text { Destination }>192.168 .5 .10</ \text { Destination }> \\
\quad<\text { SourcePort }>5000</ \text { SourcePort }> \\
\quad<\text { DestinationPort }>554<\text { /DestinationPort }> \\
\text { </ManagerRequest }>\end{array}$ \\
\hline
\end{tabular}

Figure 1. A sample manager request.

\begin{tabular}{|l|}
\hline$<$ ?xml version="1.0"? $>$ \\
$<$ ManagerResponse $>$ \\
$<$ Version $>1.0</$ Version $>$ \\
$<$ Code $>0</$ Code $>$ \\
$<$ Description $>$ Success $</$ Description $>$ \\
</ManagerResponse $>$
\end{tabular}

Figure 2. A sample manager response.

For the last part, classification rules are inserted/updated on both server's and client's routers. Then Quality of Service rules are added/modified on every routing device (router, third layer switch, firewall etc...) which are the path's members. Then Manager gives a "Go on" reply to the request. As a result of these process, an end-to-end connection path is established.

As Network Quality and Resourse Manager can set-up end-to-end connection path, it can also remove one. The removing request is also made by the server in given format Figure 1.

\section{Results}

The developed Network Quality and Resource Manager software is only tested on an isolated security camera network which includes 180 security cameras, 2 servers and various brand $2^{\text {nd }}$ and $3^{\text {rd }}$ layer switches. The testing network's basic architecture is given in Figure 3. On the test network, a simple end-to-end connection, there are at least two third layer switches.

For performance tests, a connection between the server and a random camera is created. In first round, manager is used and the connection creation time is measured. On the second round, same time measured with-out using the manager. According to the test results, the Network Quality and Resourse Manager enables the starting of the initial connection $6 \%$ faster than the normal one. Also, where there is a $0.4 \%$ packet loss on normal test, with the manager, the packet loss is reduced to $0 \%$. 


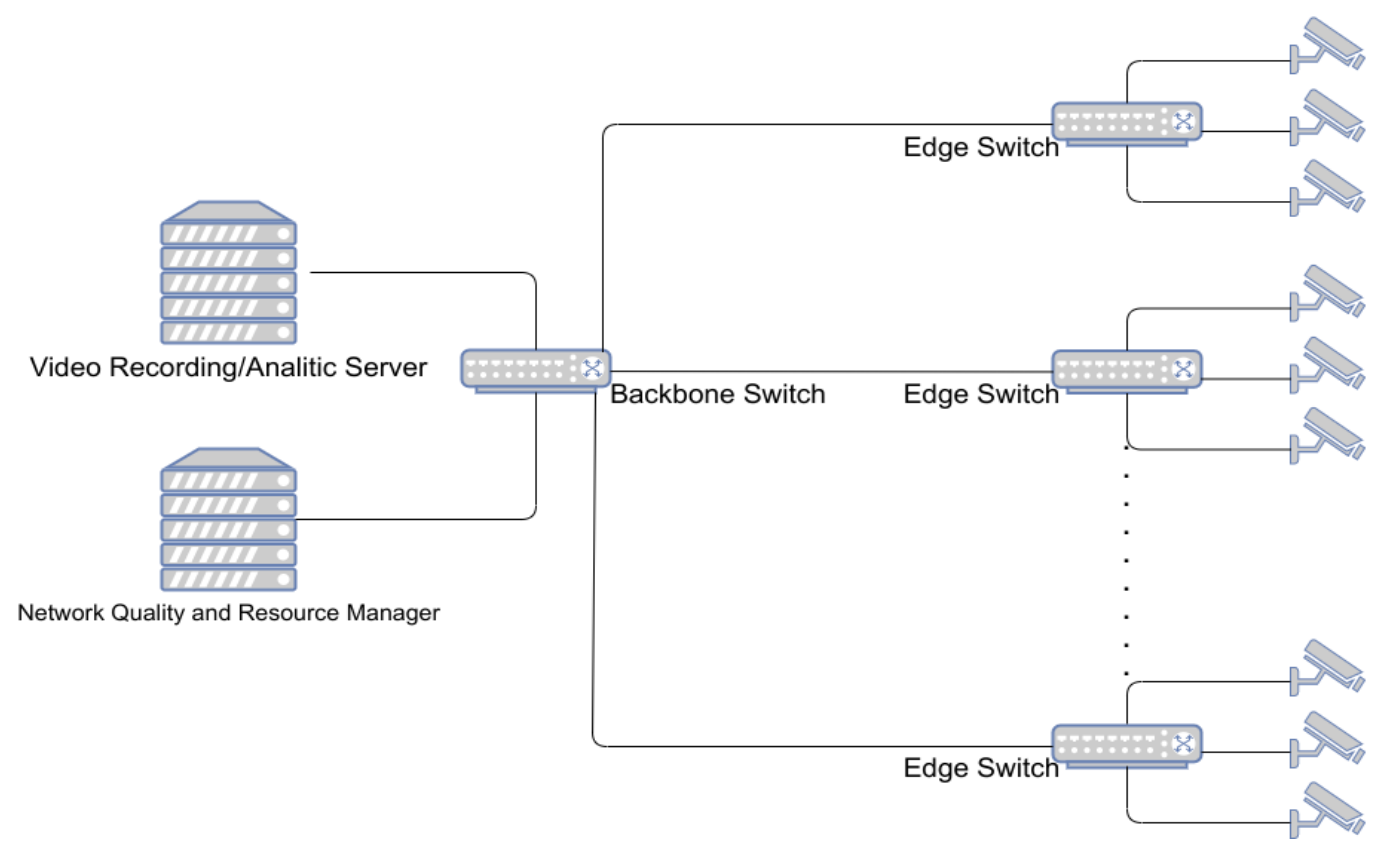

Figure 3. The network architecture.

\section{Discussion}

The first version of Network Quality and Resourse Manager lacks two sections;

First, if there is any change on the network occours, the Manager cannot detect this change. So the next versions of the manager must be able to detect the changes. The simple way to do this detection is by using the Simple Network Management Protocol's trap mechanism or spooling routing tables changes periodically.

And the second, the manager is not be able to be tested on a live network with multiple purposes. It is estimated that the manager will me more efficient with this kind of network.

\section{Conflict of interest}

The author declared that there is no conflict of interest.

\section{References}

Dostálek L, Kabelová A. 2016. Understanding TCP/IP: A clear and comprehensive guide to TCP/IP protocols. Packt Publishing Ltd, Birmingham, UK.

Prasad A, Prasad N. 2005. 802.11 WLANs and IP networking: Security, QoS, and mobility. USA MA Norwood: Artech House Inc. 